\title{
Osteocyte and osteoblast apoptosis and excessive bone deposition accompany failure of collagenase cleavage of collagen
}

\author{
Weiguang Zhao, Michael H. Byrne, Yingmin Wang, and Stephen M. Krane
}

Department of Medicine, Harvard Medical School, Medical Services (Arthritis Unit), Massachusetts General Hospital,
Boston, Massachusetts, USA

Address correspondence to: Stephen M. Krane, Arthritis Unit/Bulf 165, Massachusetts General Hospital, 55 Fruit Street, Boston, Massachusetts 02114, USA. Phone: (617) 726-2870; Fax: (617) 726-2872; E-mail: krane.stephen@mgh.harvard.edu.

Received for publication April 25, 2000, and accepted in revised form September 7, 2000.

Mice carrying a targeted mutation ( $\mathrm{r}$ ) in Col1a1, encoding a collagenase-resistant form of type I col-
lagen, have altered skeletal remodeling. In hematoxylin and eosin-stained paraffin sections, we
detect empty lacunae in osteocytes in calvariae from Col1a $1^{r / r}$ mice at age 2 weeks, increasing through
age $10-12$ months. Empty lacunae appear to result from osteocyte apoptosis, since staining of osteo-
cytes/periosteal osteoblasts with terminal deoxynucleotidyl transferase-mediated dUTP nick-end
labeling is increased in Col1a $1^{r / r}$ relative to wild-type bones. Osteocyte perilacunar matrices stained
with Ab that recognizes collagenase collagen $\alpha 1$ (I) chain cleavage ends in wild-type but not Col1a $1^{r / r}$
calvariae. Increased calvarial periosteal and tibial/femoral endosteal bone deposition was found in
Col1a ${ }^{r / r}$ mice from ages $3-12$ months. Calcein labeling of calvarial surfaces was increased in Col1a1 $1^{r / r}$
relative to wild-type mice. Daily injections of synthetic parathyroid hormone for 30 days increased
calcein-surface labeling in wild-type but caused no further increase in the already high calcein stain-
ing of Col1a $1^{r / r}$ bones. Thus, failure of collagenase cleavage of type I collagen in Col1a1 $1^{r / r}$ mice is asso-
ciated with osteocyte/osteoblast death but increases bone deposition in a manner that mimics the
parathyroid hormone-induced bone surface activation seen in wild-type mice.

J. Clin. Invest. 106:941-949 (2000).

\section{Introduction}

When bone is formed, osteoblasts at the bone surface synthesize and secrete an organic extracellular matrix (ECM) that subsequently becomes mineralized. Osteoblasts embedded in the ECM they secrete become osteocytes that form a syncytial network connected through canaliculi that permit the passage of extracellular fluid (1). Newly formed osteocytes retain several structural features of osteoblasts with an abundant and well-organized granular endoplasmic reticulum and a large Golgi region, characteristics of active protein-synthesizing cells. Osteocytes located at increasing distances from active bone-forming surfaces, however, have a scanty granular endoplasmic reticulum and a smaller Golgi apparatus. Despite their appearance as "inactive" cells, there is increasing evidence indicating that mature osteocytes are the cells that have a role in transduction of signals of mechanical loading, thereby acting as the mechano-sensors in bone $(2,3)$. The nature of these signals, which possibly include canalicular flow-induced production of prostaglandins $(3,4)$, is currently a subject of considerable interest. Although osteocytes are long-lived cells with a life span estimated at more than 20 years, some osteocytes die, as shown by the presence of empty lacunae in "inactive" bone (5, 6 ). Increased osteocyte death has been observed in specimens of iliac bone from humans with estrogen deficiency associated with the use of a gonadotropin-releas- ing hormone analogue (7) and in the bone disease induced by glucocorticoid excess (8).

Bone remodeling is thought to occur in units and is generally initiated by osteoclastic bone resorption. Although bone formation, especially during embryonic development and during postnatal periods of growth, is independent of bone resorption, during bone remodeling later in life there is evidence that responses of osteoblastic bone formation are modulated (coupled) by signals sent from osteoclasts and could involve ligands such as TGF- $\beta$ released from the ECM of bone and activated in the process of osteoclastic resorption. Locally generated ligands besides TGF- $\beta$, such as the bone morphogenetic proteins and the IGFs, also have stimulatory effects on osteoblast generation and differentiation $(9,10)$. Most recently, neural controls of bone formation, exerted through the action of leptin in the central nervous system, have also been demonstrated (11). In addition, the circulating hormone, parathyroid hormone (PTH), and the locally produced PTH-related peptide (PTHrP) act directly through the same receptor on mesenchymal cells such as osteoblasts and marrow stromal fibroblasts to modulate bone formation (12). A major action of PTH, however, is to induce bone resorption, an action exerted indirectly on osteoclasts and their precursors through direct effects on the mesenchymal cells. In stimulating bone formation, as well as resorption, 
PTH, acting on mesenchymal cells and then through direct cell-cell contacts mediated by cell-bound ligands such as osteoclast differentiation factor (RANK ligand) and/or production of soluble ligands, modulates the activity of existing osteoclasts and the differentiation of osteoclasts from precursor cells (12-15). There is evidence that osteoblastic cells exposed intermittently to relatively low concentrations of PTH function anabolically to increase bone formation, whereas osteoblastic cells exposed continuously to relatively high concentrations of PTH function catabolically to increase bone resorption $(16,17)$. The same osteoblastic cells exert these seemingly opposing responses to $\mathrm{PTH}$, probably through different intracellular pathways of signal transduction $(18,19)$. Thus, PTH in low dosages increases bone formation and bone mass in normal or ovariectomized rats $(10,16,17,20-22)$, as well as in humans with osteoporosis (23-26).

We have been examining skeletal modeling and remodeling using mice in which a mutation was targeted to Col1a1 in the region that encodes the single site in the helical domain of the type I collagen $\alpha 1$ (I) chains where collagenase cleavage occurs (27-29). The mice, termed Col1 $1^{\text {tml Jae }}$, which express the targeted mutation $(\mathrm{r})$ on both alleles $(\mathrm{r} / \mathrm{r})$ of Col1a1, produce type I collagen molecules in which neither the $\alpha 1$ (I) nor the $\alpha$ 2(I) chains are cleaved by collagenases. The Col1a $a 1^{r / r}$ mice, in contrast to wild-type mice, fail to mount an osteoclastic bone resorptive response to PTH injected subcutaneously over the calvariae (30).

We describe here further observations of the abnormal skeletal phenotype and the effects of PTH in Col1a $1^{r / r}$ mice. As early as 2 weeks of age, empty osteocyte lacunae were evident in the calvariae from Col1a1 $1^{r / r}$ mice; the number of empty lacunae increased with increasing age. Since the loss of osteocytes from their lacunae could be due to apoptosis, we used terminal deoxynucleotidyl transferase-mediated dUTP nick-end labeling (TUNEL) staining to estimate DNA strand breaks in bone cells. Many osteocytes as well as periosteal cells in Col1a $1^{r / r}$ calvariae were TUNEL positive, whereas few TUNELpositive cells were seen in wild-type calvariae. Empty osteocyte lacunae were also found in the shafts of long bones from the Col1a1 ${ }^{r / r}$ mice. We present evidence that collagenase cleavage takes place in periosteocytic ECM in wild-type but not in Col1a1 ${ }^{1 / r} \mathrm{cal}$ variae. In other cell systems, collagenase gene transcription is induced when cells bind to collagen and cleavage of the collagen with subsequent ligation through integrins (e.g., $\alpha_{v} \beta_{3}$ ) produces antiapoptotic signals $(31,32)$. We postulate that normally osteocytes (and osteoblasts) could use similar signals to maintain their viability, and, if such signals are not induced, they undergo apoptosis and their lacunae empty. We also show here that young Col1a $1^{r / r}$ mice began to develop thickening of the calvariae through deposition of new bone, predominantly at the inner periosteal surface; increased deposition of endosteal trabecular bone was found in long bones in older Col1a $1^{r / r}$ mice. The increased bone deposition in untreated Col1a1 ${ }^{r / r}$ mice was accounted for by marked activation of bone-forming surfaces, judging from the pattern of calcein labeling. This pattern in untreated Col1a $1^{r / r}$ mice resembled that in wild-type mice treated with PTH, although we have not so far detected significant differences in circulating levels of PTH in the Col1a1 ${ }^{r / r}$ compared with the wild-type mice. Thus, the failure of collagenase to cleave type I collagen in the Col1a1 ${ }^{r / r}$ mice was associated with increased osteoblast and osteocyte apoptosis, yet increased bone deposition.

\section{Methods}

Mice. In the experiments described, we used the progeny of homozygous $(r / r)$ breeding pairs of the Col1a $1^{\text {tml Jae }}$ mice $(29,30)$. Homozygous offspring had been identified by genotyping the progeny of heterozygous $\left(\right.$ Col1 $\left.a 1^{r /+}\right)$ breeding pairs using a PCR-based method (30). In these experiments, the mice referred to as wild-type controls were the wild-type progeny of Col1a $1^{r++}$ breeding pairs. The Col1a $1^{r /+}$ animals were derived from the J1/129 strain (the mutation was targeted in ES cells of this strain) and the C57BL/ 6 strain.

Tissue processing and analysis. After sacrifice by $\mathrm{CO}_{2}$ narcosis, calvariae and hind limbs were removed intact, soft tissues were gently dissected, and the bones were fixed in $10 \%$ phosphate-buffered formalin for 24 hours for further processing and analysis. After fixation, calvariae were decalcified in 14\% EDTA for 7-8 days and then dehydrated in graded alcohol. Calvariae were then bisected perpendicular to the sagittal suture through the central portion of the parietal bones, parallel to the lambdoidal and coronal sutures, and embedded in paraffin to obtain sections of a standard area. Four to six $5-\mu \mathrm{m}$-thick representative, nonconsecutive step sections were cut. The sections were routinely stained with hematoxylin and eosin (H\&E). To facilitate histomorphometric measurements, a standard length of $5 \mathrm{~mm}$ of each section from the edge of the sagittal suture to the muscle insertion at the lateral border of each bone was used. The hind limbs, after dissection of soft tissues and fixation as above, were decalcified in 14\% EDTA for 2 weeks before embedding in paraffin and sectioning. Longitudinal sections through the epiphyses of the tibias and femurs were also obtained for analysis.

Estimation of the number of osteocytes and empty osteocyte lacunae. For measurement of numbers of osteocytes and empty osteocyte lacunae in the calvariae, a standard length of $2 \mathrm{~mm}$ of each section from the edge of the sagittal suture to the muscle insertion at the lateral border of each bone was obtained for staining with $\mathrm{H} \& \mathrm{E}$. Measurements were made on digitized images using the NIH Image program (NIH, Bethesda, Maryland, USA) for the Macintosh computer. The area in square millimeter per millimeter of standard length, the total number of empty osteocyte lacunae per 
square millimeter, and the percentage of empty lacunae per total lacunae were calculated.

Identification of apoptotic cells. The TUNEL method was used (33) to detect apoptotic cells in 5-mm-thick sections of paraffin-embedded tissue using the kit obtained from Boehringer Mannheim Biochemicals Inc. (Indianapolis, Indiana, USA). Fluorescence microscopy with an inciting wavelength of $485 \mathrm{~nm}$ and an analyzing wavelength of $510 \mathrm{~nm}$ was used to identify the green-fluorescing cells. Numbers of TUNELstained cells in calvarial sections of standard length were counted using digitized images as described above.

Assessment of collagenase cleavage in tissue sections. A mouse mAb, 9A4, was generated (34) using the synthetic peptide Gly Pro Pro Gly Pro Gln Gly linked to keyhole limpet hemocyanin as the immunogen and was generously provided by Ivan Otterness (Central Research Division, Pfizer, Groton, Connecticut, USA). This sequence of amino acid residues 768-775 in the $\alpha 1$ (II) chains precedes the collagenase-cleavage site between Gly775 and Leu776 in $\alpha 1$ (II) chains $(34,35)$. Whereas mAb 9A4 also recognizes the heptapeptide sequence Gly Thr Pro Gly Pro Gln Gly in the COOHterminal end of the larger, three-quarter fragment (termed $\mathrm{A}^{\alpha 1[\mathrm{I}]}$ or $\left.\mathrm{TC}^{\mathrm{A} \alpha 1[\mathrm{I}]}\right)$ of the $\alpha 1(\mathrm{I})$ chain of type I collagen, it does not recognize this sequence in uncleaved, denatured type I or type II collagen. To use the $\mathrm{Ab}$ for assessment of collagenase cleavage of type I collagen in mouse tissue sections, yet maintain a low background of staining, the Ab was biotinylated, and binding of $\mathrm{Ab}$ to epitope was assayed using an avidinlinked peroxidase system (Vector Laboratories, Burlingame, California, USA). Controls comprised samples with the avidin-peroxidase but without $\mathrm{Ab}$.

Calcein labeling. To further define the abnormal bone growth in the Col1a1 ${ }^{r / r}$ mice, a modified histomorphometric analysis was performed using double labeling with calcein as the marker. Calcein $(25 \mathrm{mg} / \mathrm{kg}$; Sigma Chemical Co., St. Louis, Missouri, USA) was injected intraperitoneally twice at intervals of 11 days, and the mice were sacrificed 4 days after the last injection. Calcein labeling in the calvariae was assessed using undecalcified, frozen, 5-mm-thick sections of tissue fixed previously in formalin. Calcein labeling of long bones was assessed using formalin-fixed, undecalcified samples embedded in methyl methacrylate, and 5-mm-thick sections were prepared. A Nikon microscope was used and the pattern of fluorescence analyzed with an inciting wavelength of $485 \mathrm{~nm}$ and an analyzing wavelength of $510 \mathrm{~nm}$.

Effects of PTH in vivo. To measure anabolic responses of bone to low-dose systemic PTH in vivo, we followed the protocols developed by Hock and coworkers $(16,17)$. Synthetic human PTH(1-34) was obtained from A. Khatri (Endocrine Unit, Massachusetts General Hospital, Boston, Massachusetts, USA) and was dissolved in vehicle (1 mM HCl, 0.1\% BSA). To measure the effects of PTH on activation of bone surfaces, 3-month-old mice were injected once daily, intraperitoneally, for 30 days with vehicle or synthetic hPTH 1-34, $40 \mu \mathrm{g} / \mathrm{kg}$, and with calcein, $25 \mathrm{mg} / \mathrm{kg}$, intraperitoneally, the latter at 15 and 26 days. The mice were then sacrificed at 30 days.

Quantification of bone area and active bone surfaces. For the calvarial sections, the total areas of bone and marrow cavities (i.e., bone and marrow between the inner and outer periosteal surfaces within the 5 - $\mathrm{mm}$ length from the sagittal suture) were measured on digitized images using the NIH Image program for the Macintosh computer. To reduce measurement errors, at least two of the most central sections among all sections from each sample were examined and quantified. Each area of bone per standard length was digitized twice using the NIH Image program, and two readings were averaged. All measured areas of bone within each section were then summed. The amount of inner and outer bone surfaces labeled with calcein and the distances between the labels were similarly quantified. Statistical significance was determined using ANOVA.

Blood measurements. Blood ionized calcium concentration $\left[\mathrm{Ca}^{2+}\right]$ was determined with the 634 ISC $\mathrm{Ca}^{++} / \mathrm{pH}$ Analyzer from Ciba Corning Diagnostics (Medfield, Massachusetts, USA). Serum PTH levels were measured using the Rat IRMA PTH kit (Immunotopics Inc., San Clemente, California, USA). To obtain sufficient serum required for this assay, blood was obtained after sacrifice and serum separated. Measurements were made on $200-400 \mu \mathrm{l}$ of serum. Grossly hemolyzed samples were not analyzed.

\section{Results}

Osteocytes and empty osteocyte lacunae in Col1a1 ${ }^{r / r}$ mice. We first noted the presence of significant increases in the number of empty osteocyte lacunae in 4-week-old Col1a1 ${ }^{r / r}$ mice compared with 4-week-old wild-type mice, as shown in representative sections of calvariae stained with H\&E in Figure 1a. These changes persisted in calvariae from 10 to 12 -month-old Col1a1 ${ }^{r / r}$ mice (Figure 1b). The calvariae in the older Col1a $1^{r / r}$ mice were also thicker than those of the wild-type mice (see below). As shown in Figure 1b, although the area of the calvariae was comparable in 4-week-old wild-type and Col1a ${ }^{r / r}$ mice, the number of empty lacunae was increased approximately tenfold in the Col1a $1^{r / r}$ mice compared with the wild-type mice. In 4-week-old wild-type mice $(n=8)$, there were $12 \pm 2$ (SEM) empty lacunae per $\mathrm{mm}^{2}$ $\left(1.8 \pm 0.32 \%\right.$ of total), whereas in 4 -week-old Col1a $1^{r / r}$ mice $(n=8)$, there were $120 \pm 19$ empty lacunae per $\mathrm{mm}^{2}$ (15 $\pm 2.5 \%$ of total). In 10 - to 12 -month-old wild-type mice $(n=5)$, there were $15 \pm 5.6$ empty lacunae per $\mathrm{mm}^{2}$ ( $3 \pm 1.2 \%$ of total), whereas in 10 - to 12 -month-old Col1a ${ }^{r / r}$ mice $(n=7)$, there were $150 \pm 18$ empty lacunae per $\mathrm{mm}^{2}(22 \pm 2.4 \%$ of total). Increased numbers of empty osteocyte lacunae were seen in Col1a $1^{r / r}$ mice as young as 2 weeks of age, but in calvariae from neonatal wild-type and Col1a $1^{r / r}$ mice all osteocyte lacunae were filled (data not shown). Empty osteocyte lacunae were also found in tibias and femurs, the long bones examined in the Col1a1 ${ }^{r / r}$ mice (data not shown). 
Apoptosis in Col1a1 $1^{r / r}$ mouse calvariae. In view of the remarkable increase in the number of empty osteocyte lacunae in the calvariae from the Col1a $1^{r / r}$ mice, we tested for the occurrence of apoptosis in cells that filled the remaining osteocyte lacunae, using TUNEL staining. It can be seen in the example from 4-week-old mice shown in Figure 2a that the number of persisting TUNEL-positive osteocytes in the calvariae from both 4-week-old and 10-month-old Col1a1 ${ }^{r / r}$ mice was increased, compared with calvariae from wild-type mice of comparable age. TUNEL staining was also observed in cells in the periosteum of the Col1a $1^{r / r}$ mice (Figure 2a), which included osteoblasts, preosteoblasts, or stromal fibroblasts, but they were not further identified. In the calvariae from the wild-type mice, however, TUNEL staining was observed only in rare, scattered bone cells of all types. Increased TUNEL staining was also seen in the calvariae from 2 -week-old Col1a $1^{r / r}$ mice but not in osteoblasts or osteocytes in bones from neonatal Col1a $1^{r / r}$ or wild-type mice (data not shown). As shown

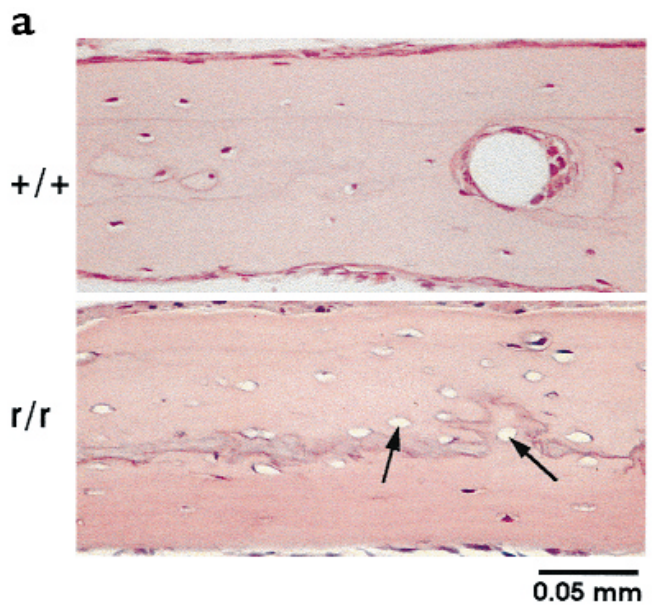

b

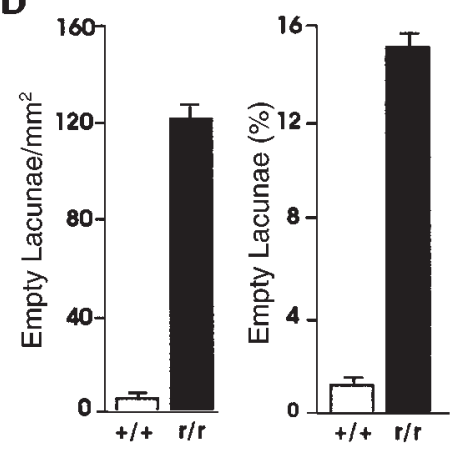

Figure 1

Presence of empty osteocyte lacunae in calvariae from wild-type and Col1a1r/r mice. (a) Sections of calvariae from 4-week-old wild-type and Col1a $1^{r / r}$ mice stained with H\&E. Note some of the empty osteocyte lacunae (arrows) in the sections from the Col1a $11^{r / r}$ mice; all osteocyte lacunae are filled with cells in the section shown from the wild-type mice. (b) Quantification of empty osteocyte lacunae in calvariae from 4- to 6-week-old wild-type and Col1a 1 r/r mice. The number of empty lacunae per $\mathrm{mm}^{2}$ standard area is shown in the left graph and the percentage of empty lacunae per total lacunae is shown in the right graph. in Figure 2b, in calvariae from 4 week-old wild-type mice, $7.3 \pm 1.0$ (SEM) apoptotic osteocytes per $\mathrm{mm}^{2}$ were counted, whereas in calvariae from Col1a $1^{r / r}$ mice, 73.8 $\pm 7.4(\mathrm{SEM})(n=5)$ apoptotic osteocytes per $\mathrm{mm}^{2}$ were counted. In each of the two 8-month-old wild-type mice sampled, 9 and 7 apoptotic osteocytes per $\mathrm{mm}^{2}$ were counted, whereas in four Col1a1 $1^{r / r}$ mice a mean of $83.0 \pm$ 2.7 (SEM) apoptotic osteocytes per $\mathrm{mm}^{2}$ were counted. Collagenase cleavage in the perilacunar osteocyte ECM. Since the only defect engineered in the Col1a $1^{r / r}$ mice was alteration of amino acids around the collagenasecleavage site in $\alpha 1$ (I) chains that conferred resistance to cleavage, we asked whether osteocytes normally release collagenase that cleaves type I collagen in the surrounding ECM. To pursue the answer, we used the biotinylated mAb, 9A4 (34), that recognizes the unique amino acid sequence epitope in the $\mathrm{COOH}-$ terminal end of the three-quarter-length fragment of the $\alpha 1(\mathrm{I})$ chain of type I collagen cleaved by collagenases. It is shown in Figure 3 that staining of scattered osteocytes and their surrounding ECM was present in the calvariae from wild-type mice, but not in calvariae from Col1a1 ${ }^{r / r}$ mice. No staining was detected using avidin-peroxidase alone in the absence of mAb 9A4. These findings are consistent with the production by wild-type osteocytes of collagenase that can cleave type I collagen in the perilacunar ECM. The absence of staining of the perilacunar ECM with mAb 9A4 in Col1a $1^{r / r}$ mice provides in vivo observations consistent with our previous in vitro observations $(29,36)$ that Col1a ${ }^{r / r}$ type I collagen is not cleaved at the helical locus by collagenases.

Calvarial bone area in Col1a1 $1^{r / r}$ and wild-type mice. We observed no differences in the thickness of the calvariae in Col1a1 ${ }^{r / r}$ mice compared with wild-type mice younger than 4 weeks of age. In Col1a ${ }^{r / r}$ mice older than approximately 6 weeks of age, however, we began to detect increases in calvarial thickness. In Figure 4a are shown representative histological sections of calvariae stained with $\mathrm{H} \& \mathrm{E}$ from wild-type and Col1a1 ${ }^{r / r}$ mice at 4 weeks and 12 months of age. Whereas calvarial thickness was similar in 4-week-old wild-type and Col1a1 ${ }^{r / r}$ mice, calvarial thickness in 12-month-old Col1a ${ }^{r / r}$ mice was markedly increased compared with that of 12-month-old wild-type mice. In addition, the inner periosteal surface appeared irregular and wavy in Col1a1 ${ }^{r / r}$ calvariae, in contrast to the smooth inner periosteal surface of wild-type calvariae. Calvarial thickness was quantified in Col1 $a 1^{r / r}$ and wild-type mice at different ages using a standard length of $5 \mathrm{~mm}$, as shown in the bar graph in Figure 4b. Although calvarial thickness did not differ in wild-type and Col1a1 $1^{r / r}$ mice at 4 weeks of age, at 12 months of age, calvarial thickness in Col1a1 ${ }^{r / r}$ mice was approximately twice that in wild-type mice $(P<0.0001)$.

Measurements of active bone-formation surfaces and effects of $P T H$. To determine if the increase in calvarial thickness in older Col1a1 ${ }^{r / r}$ mice could be due to increased bone formation, we used in vivo labeling with calcein as a 


\section{Figure 2}

Fluorescence photomicrographs of sections of calvariae from 4-week-old wildtype and Col1a1r/r mice stained by the TUNEL method. (a) Presence of TUNELpositive cells in calvariae from wild-type and Col1a1r/r mice. Open bars indicate outer (top) and inner (bottom) surfaces of the periosteum. (b) Quantification of TUNEL-positive cells in calvariae from wild-type and Col1a1r/r mice.
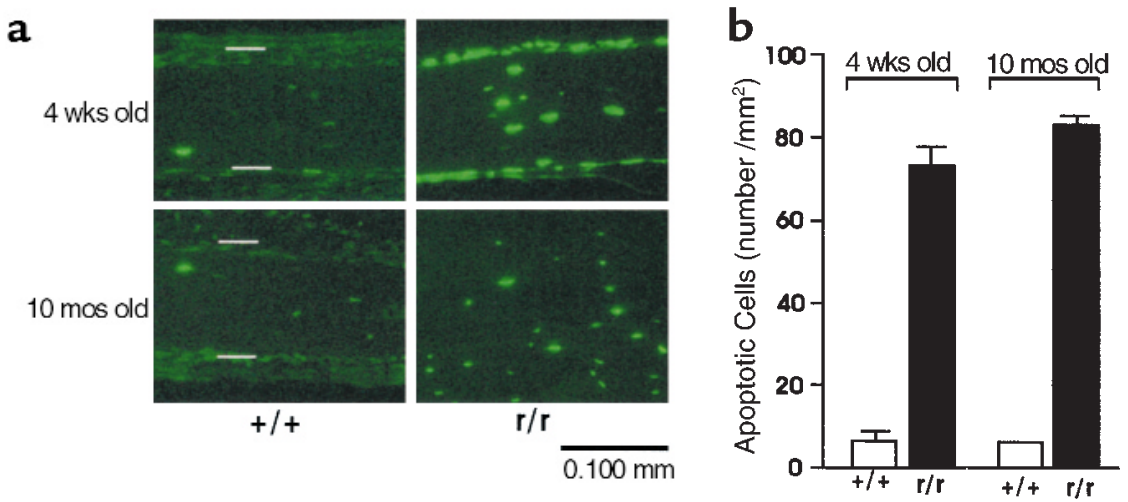

marker in animals 3 months of age or older. As shown in the examples in Figure 5a, in calvariae from untreated (vehicle alone) wild-type mice, most of the fluorescent labels were observed inside the bone marrow cavity. There was rarely labeling of the inner periosteal surfaces and only occasionally labeling at the outer periosteal surfaces. In striking contrast, however, marked and extensive double labeling was evident at both periosteal surfaces in untreated Col1a1 ${ }^{r / r}$ mice (Figure 5a). Since PTH in low doses has been demonstrated to have anabolic effects on bone, and we have shown that catabolic effects of high doses of PTH on bone resorption are markedly blunted in the Colla $1^{r / r}$ mice (30), we designed experiments to compare the anabolic effects of low-dose PTH in the wild-type and Col1 $11^{r / r}$ mice (see protocol in Methods). PTH treatment increased calcein labeling, particularly evident at both periosteal surfaces. These differences were quantified as shown in Figure $5 \mathrm{~b}$. There was marked activity at both periosteal surfaces in Col1a $1^{r / r}$ mice with or without PTH treatment. In contrast, there was little labeling of either surface in wild-type mice treated with vehicle, but in wild-type mice treated with PTH, labeling was markedly increased at both periosteal surfaces. There was a parallel increase in the mineral apposition rate measured as the distance between the two calcein labels divided by the time interval between injections in wild-type mice treated with PTH (Figure 5c). The spontaneous mineral apposition rate tended to be greater at the inner compared with the outer periosteal surface in Col1a1 ${ }^{r / r}$ mice.

Excessive endosteal bone in femurs from Col1a1 ${ }^{r / r}$ mice. Changes were also consistently observed in the long bones from Col1a ${ }^{r / r}$ mice. Examples shown in Figure 6a are longitudinal, histological sections of portions of the midfemoral shaft from 10-month-old mice. The endosteal surface was smooth in wild-type mice, with little deposition of new endosteal bone. The endosteal surface in Col1a1 ${ }^{r / r}$ mice, however, contained abundant, new, endocortical trabecular bone surrounding active marrow spaces. Total bone (cortical plus trabecular) in this region of the diaphysis was greater in all samples examined from Col1a1 ${ }^{r / r}(n=6)$ compared with wildtype mice of similar age $(n=6)$. The pattern of calcein labeling in the long bones from untreated wild-type and Col1a1 $1^{r / r}$ mice also differed markedly as shown in
Figure 6b. In Col1a1 $1^{r / r}$ mice, calcein labeling was observed at both periosteal and endosteal surfaces with most of the fluorescence in the new bone at the endosteal surface. Only limited and scattered labeling was found on the endosteal surfaces of the femurs and tibias from wild-type mice.

Blood ionized-calcium and PTH levels. Since we showed in these experiments that PTH activates bone surfaces, and we had shown previously that the resorptive effects of PTH are blunted in the Col1a ${ }^{r / r}$ mice, we asked whether spontaneous secondary hyperparathyroidism was present in the Col1a1 $1^{r / r}$ mice. Levels of ionized calcium were not different in the Col1a $1^{r / r}$ compared with the wild-type mice (data not shown). Although the mean levels of PTH were higher in Col1a1 ${ }^{r / r}$ compared with wild-type mice $(4.7 \pm 0.6$ $[\mathrm{SEM}] \mathrm{pg} / \mathrm{ml}, n=15$, versus $3.7 \pm 0.2[\mathrm{SEM}] \mathrm{pg} / \mathrm{ml}$, $n=17$ ), the differences were not significant.

\section{Discussion}

Although the homozygous (Col1a $1^{r / r}$ ) mice had normal development of the appendicular skeleton during embryogenesis and during the first week after birth, after approximately 2 weeks of age, aspects of the skeletal phenotype became apparent. There was detectable

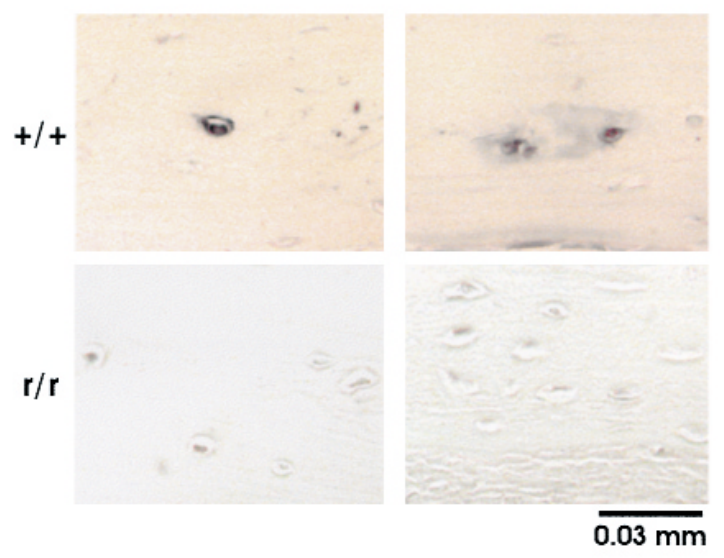

Figure 3

Photomicrographs of calvarial sections from 4-week-old wild-type and Col1a $1^{r / r}$ mice stained with $9 \mathrm{~A} 4 \mathrm{mAb}$ to the collagenase cleavage epitope. Two separate representative areas of sections from different mice are shown. No counterstain was used. 
loss of osteocytes from their lacunae in calvariae and long bones of Col1a $1^{r / r}$ mice older than 2 weeks, and this loss continued as they aged. Beginning at approximately 2 weeks of age, apoptosis was detected using TUNEL staining in a significant number of periosteal osteoblasts and in persisting osteocytes in Col1a $1^{r / r}$ mice. Despite bone cell apoptosis, Col1a1 ${ }^{r / r}$ mice after approximately 4 weeks of age began to deposit excessive bone at endosteal surfaces in long bones and in calvariae predominantly at the inner periosteal surface adjacent to the dura mater; calvarial thickness nearly doubled by approximately 10 months of age. It had been shown that PTH injected subcutaneously over the calvariae induced bone resorption at intracortical locations in the calvariae, but not at the outer and inner periosteal surfaces (37). Thus, the new bone formation observed here spontaneously in the Col1a ${ }^{r / r}$ mice was appositional and could not be accounted for by decreased bone resorption alone. In the long bones, the extra bone was also deposited in the endosteal region of the shafts in positions normally occupied by marrow elements and in this sense could also be considered appositional. Furthermore, the extra bone was deposited on active surfaces labeled with calcein in vivo. In calvariae from untreated Col1a $1^{r / r}$ mice, almost all of the surfaces were labeled with calcein, whereas there was minimal calcein label in calvariae from untreated wildtype mice. In the bone shafts the endosteal bone was also intensely labeled with calcein. Nevertheless, PTH administered for 30 days increased calcein labeling in wild-type mice to an extent nearly that observed spontaneously in Col1a1 ${ }^{r / r}$ mice.

It was first shown in 1964 that administration of parathyroid extract to mice increased collagenolytic activity in organ cultures of bone removed from these animals (38). Later, it was found that PTH added in vitro to bone organ cultures had a similar stimulatory effect (39). Subsequently, this collagenase was cloned $(40,41)$ and identified as MMP-13 (42-43), and it was demonstrated that its gene expression in cultured osteoblast-like cells could be stimulated by PTH (40, $44,45)$. In the developing mouse skeleton, MMP-13 is expressed in distal hypertrophic chondrocytes of the growth plate and in osteoblasts in the shafts of the long bones (46-50). In mice with a null mutation in the $\mathrm{PTH} / \mathrm{PTHrP}$ receptor gene, expression of MMP-13 in the distal growth plate and in the primary center of ossification of late embryos is markedly reduced (50). Other MMPs that could function potentially as collagenases, besides MMP-13, are expressed in skeletal cells during development, including MMP-8 (neutrophil collagenase, collagenase-2) (51) and MT1-MMP (52). MMP-1 is expressed in human skeletal cells, but the presence of the orthologue of MMP-1 has not been reported in mice or rats. Although the soluble form of MT-1MMP (53) has not yet been tested by us, the collagenases other than MMP-13 also do not cleave the Col1a ${ }^{r / r}$ collagen at the helical site. In two reports of the phenotype of MT1-MMP-null mice $(54,55)$, there is no description of skeletal abnormalities of the type described here. Since these changes develop in the Col1a ${ }^{r / r}$ mice only after approximately 4 weeks of age, it is not yet possible to determine if similar abnormalities would be seen in the MT1-MMP-/- mice, since most of these animals die by 3 weeks of age.

To relate the apoptosis of osteocytes to the targeted defect in collagenase cleavage of type I collagen (the major fibrillar organic component of bone) in the Col1a1 ${ }^{r / r}$ mice, it was essential to demonstrate that osteocytes produced collagenase. We were successful using the mAb, 9A4 (34), in detecting the specific epitope produced by collagenase cleavage in the periosteocytic ECM in wild-type mice but not in Col1a1 $1^{r / r}$ mice. These observations provide further evidence that the Col1a $1^{r / r}$ collagen, which is not cleaved by collagenases in vitro, is not cleaved by collagenases in vivo. We had shown previously that MMP-13, but not other collagenases, makes an additional cleavage in the $\mathrm{N}$ telopeptide region of type I collagen in vitro $(29,36)$. It has not yet been possible, however, to demonstrate

\footnotetext{
Figure 4

The thickness of calvariae in Col1a $1{ }^{r / r}$ mice increases with increasing age. (a) Histological sections of calvariae from wild-type and Col1a $1^{r / r}$ mice at 4 weeks and 12 months of age, cut perpendicularly to the sagittal suture and stained with H\&E. The outer periosteal surfaces of each section are at the top and the inner periosteal surfaces are at the bottom. The thickness of the calvariae was similar in 4-week-old wild-type and Col1a $1{ }^{r / r}$ mice, but the thickness in 12-month-old Col1a1r/r mice was markedly increased, compared with wildtype mice. (b) Quantification of calvarial bone area in ten wild-type and ten Col1a 1 r/r mice ( 4 weeks old) and five wild-type and seven Col1a1 ${ }^{r / r}$ mice (10 months old).
}
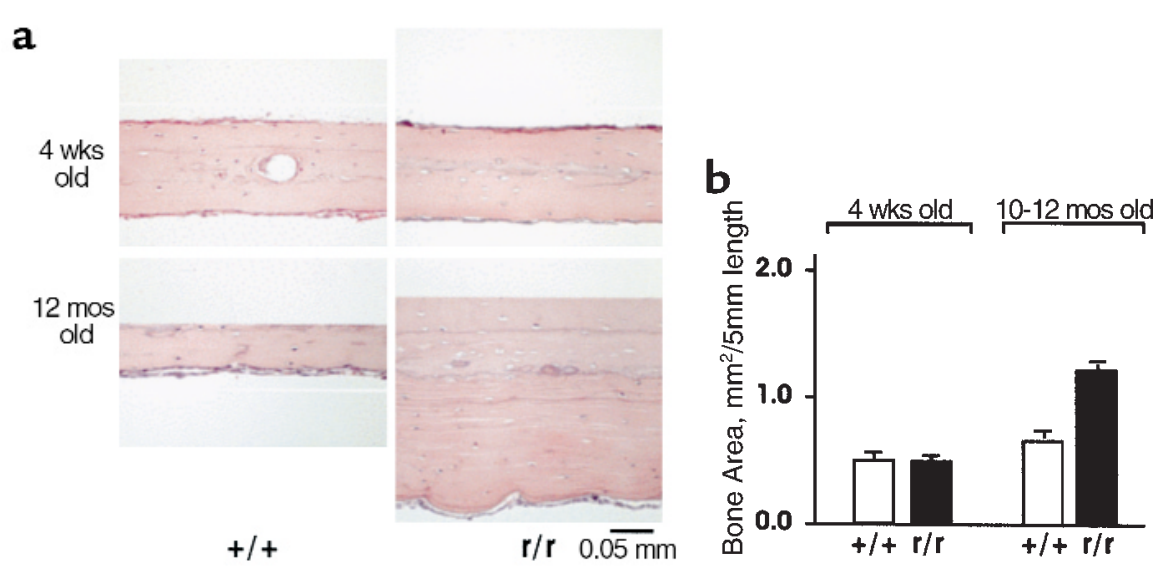

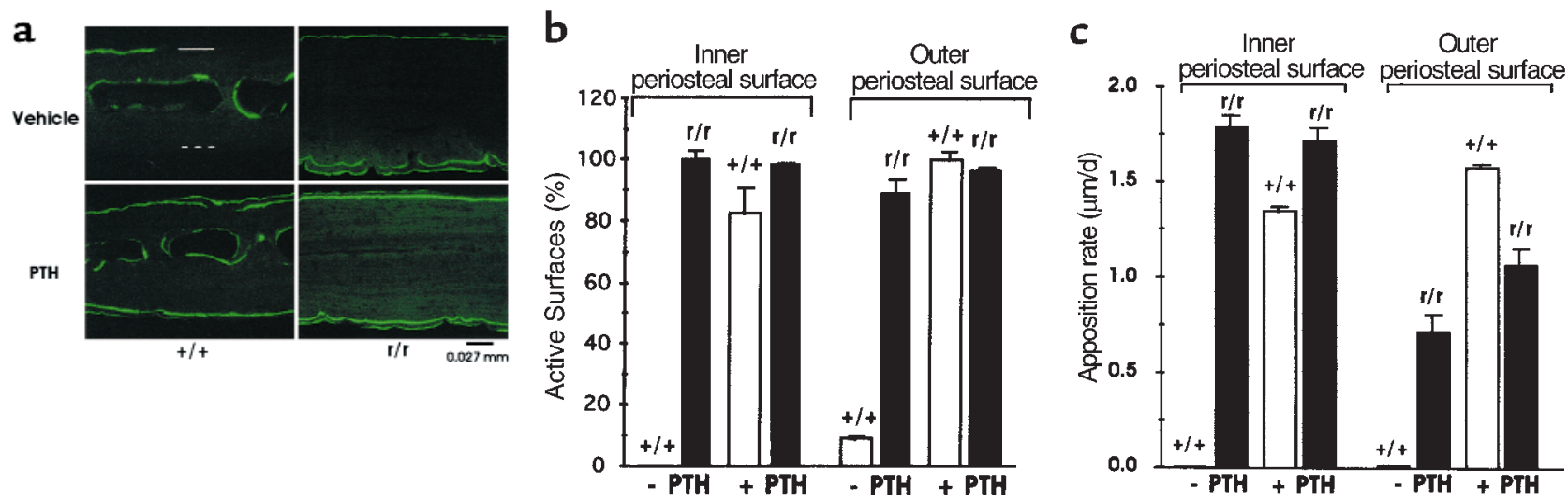

Figure 5

Calcein labeling of bone surfaces from 3-month-old wild-type and Col1a $1^{r / r}$ mice. (a) Frozen sections of calvariae, examined by fluorescence microscopy, from mice given vehicle alone or PTH daily and injected with single doses of calcein at 1 and 11 days before sacrifice (see Methods). The outer periosteal surface in the sample from the vehicle-treated wild-type mouse is indicated by the solid open bar and the inner surface by the dashed open bar. In this section of calvariae from the untreated wild-type mice, the fluorescent calcein label was limited to inside the bone marrow cavity; only scattered label was seen occasionally at the outer periosteal surface. In the Col1a $1 r / r$ mice, marked and extensive labeling was evident at both periosteal surfaces. Note that the increased calvarial thickness in the 3-month-old Col1a 1 r/r mice. In the PTH-treated wild-type mice, there was marked calcein labeling at both surfaces. The calcein labeling, already extensive in the untreated Col1a1r/r mice, was not further increased by PTH. (b) Quantification of calcein labeling of active calvarial surfaces. (c) Quantification of bone apposition rate on active calvarial surfaces.

whether the $\mathrm{N}$-telopeptide cleavage takes place in vivo, and since the Col1a1 ${ }^{r / r}$ collagen can be cleaved by MMP13 at the $\mathrm{N}$-telopeptide site, the latter cleavage would not affect the phenotype described.

It has been emphasized by Werb (56) that the ECM provides adhesive signals that control cell viability and that degradation of the ECM is the most effective mechanism for altering cellular anchorage in vivo. We speculate that osteocytes, through the release of collagenase into the surrounding ECM, normally generate a survival signal. This signal would be present in the wild-type mice but absent or diminished in the Col1a $1^{r / r}$ mice. Some evidence to support this hypothesis has been obtained from work showing that cells can bind to type I collagen through the $\alpha_{2} \beta_{1}$ integrin (57) and that following ligation of $\alpha_{2} \beta_{1}$, procollagenase gene transcription is induced with secretion and activation of the procollagenase (58). It has been demonstrated, e.g., in melanoma cells, that after collagenase cleavage of type I collagen and further unwinding of the chain ends, a cryptic binding site(s) is revealed for the $\alpha_{v} \beta_{3}$ integrin $(31,32)$. Normally, binding of the $\alpha_{v} \beta_{3}$ integrin to this cryptic site(s) could then promote some adhesion-dependent survival signal necessary for cells to progress normally through the cell cycle. An important regulator of cell-cycle arrest and apoptosis is the tumor suppressor gene, p53. Failure to bind through $\alpha_{v} \beta_{3}$ in endothelial cells or melanoma cells results in apoptosis, possibly mediated by $\mathrm{p} 53$ and $\mathrm{p} 21^{\mathrm{WAF} 1 / \mathrm{CIP} 1}$, a cyclindependent kinase inhibitor $(39,59,60)$. There are other possible explanations for the osteocyte death in Col1a1 ${ }^{r / r}$ mice. There is evidence, for example, that bone ECM is a storage source of growth factors that are released after its degradation $(56,61,62)$. Some factor present in the periosteocytic extracellular matrix not released because of failure of collagenase to act on type I collagen might also be critical for cell survival.

It should also be considered that the effects observed in Col1a $1^{r / r}$ mice could be related to the presence of the mutant collagen per se, independent of resistance to the action of collagenase. In the mutation that we targeted (27) to the Colla1 gene that induces resistance to collagenase cleavage, both Gln774 and Ala777 (in the -Y- position of the -Gly-XY- triplet) are replaced with Pro residues that would probably be 4-hydroxylated to hydroxyprolines (Hyp) in a region of the molecule that is relatively poor in Pro residues in the -Y- position (35). Thus, the presence of these additional Hyp residues would probably further stabilize the collagen triple helix and inhibit unfolding of the triple helix at this locus (35). The increased stability of the collagen helix that contributes to the mechanism of collagenase resistance of the mutant collagen might also alter its binding to other ECM components, as well as to cells.

We raise the possibility that osteocyte apoptosis could play a role in the deposition of excessive bone observed in Col1a1 ${ }^{r / r}$ mice. Responses of bone to mechanical stress that result in changes in mass and structure are mediated by osteocytes, the predominant cells of adult bone tissue (63). Osteocytes appear to act as mechano-sensors, and the flow of fluid through the lacuno-canalicular network that connects these cells with the bone-lining cells provides the stress-derived mechanical signals. Spontaneously in the Col1a $1^{r / r}$ mice there was as much as a tenfold increase in the number 


\section{Figure 6}

Presence of excessive endosteal bone in femurs from Col1a $1^{r / r}$ mice. (a) Longitudinal histological sections stained with $\mathrm{H} \& \mathrm{E}$ of femurs from 10month-old wild-type and Col1a $1^{r / r}$ mice. The location of the periosteal surface (black and white arrows) and endosteal surface (black arrows) is indicated. In wild-type mice the endosteal surface was smooth, and there was no evident endosteal new bone. In Col1a $1^{r / r}$ mice, in contrast, abundant endocortical new bone covered the endosteal surface. (b) Sections of femurs, embedded in methyl methacrylate and examined by fluorescence microscopy, from 10-month-old wild-type and Col1a $1^{r / r}$ mice injected twice with calcein, 30 days apart. Bo, bone; Ma, marrow area. The periosteal surface is indicated by the solid open bar and the endosteal surface by the dashed open bar. Note minimal calcein label at the endosteal surface of the femur from the wild-type mouse in contrast to the striking labeling of the new endosteal bone (a) in the femur from the Col1a $1^{r / r}$ mouse.

of apoptotic osteoblasts and osteocytes and a parallel increase in the number of empty osteocyte lacunae; indeed, in 10-month-old Col1a $1^{r / r}$ mice more than one fifth of the osteocyte lacunae were empty. The new bone formation took place despite the depletion of viable osteocytes. Since no signal could pass over the lacuno-canalicular network to osteoblasts at bone surfaces from empty osteocyte lacunae, we speculate that the normal osteocyte $\rightarrow$ osteoblast signal could be an inhibitory one; loss of the osteocyte signal could then lead to increased surface osteoblast activity.

Although spontaneously in wild-type mice there was little calcein labeling of calvarial surfaces, in PTHtreated wild-type mice there were marked increases in calcein-surface labeling that approached that of untreated Col1 $11^{r / r}$ mice; no further effect of PTH was observed in Col1a $1^{r / r}$ mice since bone formation was presumably already maximal. Although so far we have not detected significant differences in circulating levels of PTH in the Col1a1r/r compared with the wildtype mice, low-grade hyperparathyroidism, secondary to decreased bone resorption in the presence of resistance to collagenase cleavage of type I collagen, might still account for activation of bone-forming surfaces in Col1a $1^{r / r}$ mice. The observations of Jilka et al. (64) should be considered in this regard. They showed that the increase in bone mass that accompanies the administration of PTH to normal or osteopenic mice results not from osteoblastogenesis, but from prevention of osteoblast apoptosis. We raise the possibility that this antiapoptotic signal could normally be mediated by the action of PTH in stimulating expression of collagenase. The high rate of apoptosis in the Col1a $1^{r / r}$ mice might thus be ascribable to failure to cleave type I collagen, thereby blocking access of integrins to the cryptic epitope in the cleaved chains and abrogating an antiapoptotic signal. In the Col1a $1^{r / r}$ animals there was excessive deposition of bone despite increased apoptosis of some osteoblasts. Antiapoptotic effects of PTH might be dissociated from those on activation of bone-forming surfaces.
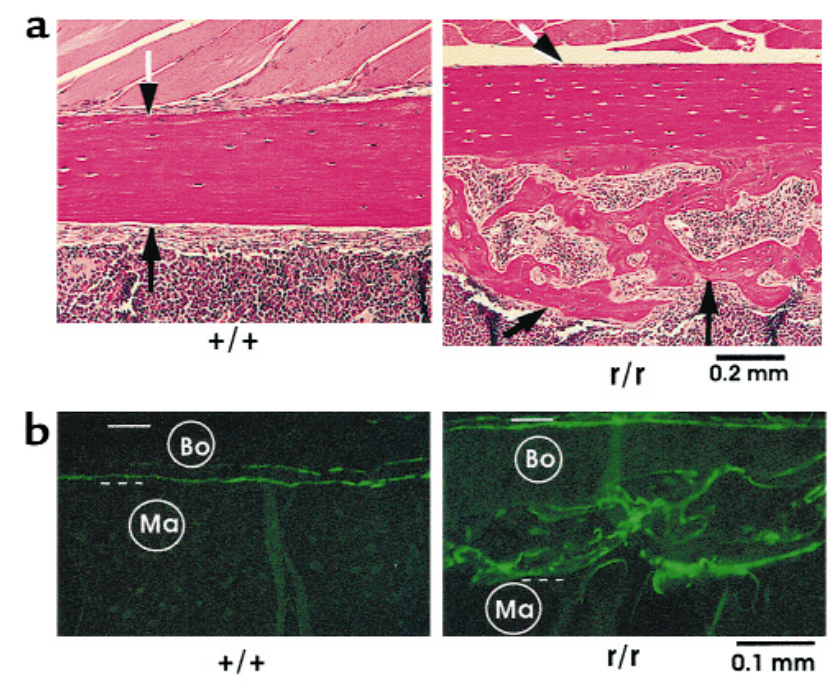

Whatever the detailed mechanisms, these observations in the collagenase-resistant Col1 a $1^{\mathrm{tml} J a e}$ mice lead us to propose a role for collagenolysis mediated by MMPs in maintaining the viability of bone cells in the postnatal skeleton. Whether or not osteocyte apoptosis observed in osteoporosis due to estrogen deficiency (7) or glucocorticoid excess (8) in humans could also involve interruption of cell/matrix interactions as a consequence of altered proteolysis remains to be determined.

\section{Acknowledgments}

This work was supported by grants from the NIH: AR03564, AR-07258, AR-44855, and AR-44815.

1. Doty, S.B., Robinson, R.A., and Schofield, B. 1976. Morphology of bone and histochemical staining characteristics of bone cells. In Handbook of physiology. R.O. Greep, E.B. Astwood, and G.D. Aurbach, editors. American Physiological Society. Washington, DC, USA. 3-23.

2. Mullender, M.G., and Huiskes, R. 1997. Osteocytes and bone lining cells: which are the best candidates for mechano-sensors in cancellous bone? Bone. 20:527-532.

3. Burger, E.H., and Klein-Nulend, J. 1999. Mechanotransduction in bonerole of the lacuno-canalicular network. FASEB J. 13(Suppl.):S101-S112.

4. Ajubi, N.E., Klein-Nulend, J., Alblas, M.J., Burger, E.H., and Nijweide, P.J. 1999. Signal transduction pathways involved in fluid flow-induced $\mathrm{PGE}_{2}$ production by cultured osteocytes. Am. J. Physiol. 276:E171-E178.

5. Frost, H.M. 1960. In vivo osteocyte death. J. Bone Joint Surg. 42A:138-143.

6. Dunstan, C.R., Somers, N.M., and Evans, R.A. 1993. Osteocyte death and hip fractures. Calcif. Tiss. Int. 53(Suppl. 1):S113-S117.

7. Tomkinson, A., Reeve, J., Shaw, R.W., and Noble, B.S. 1997. The death of osteocytes via apoptosis accompanies estrogen withdrawal in human bone. J. Clin. Endocrinol. Metab. 82:3128-3135.

8. Weinstein, R.S., Jilka, R.L., Parfitt, A.M., and Manolagas, S.C. 1998. Inhibition of osteoblastogenesis and promotion of apoptosis by glucocorticoids. J. Clin. Invest. 102:274-282.

9. Rodan, G.A., and Harada, S. 1997. The missing bone. Cell. 89:677-680.

10. Rodan, G.A. 1998. Control of bone formation and resorption: biological and clinical perspective. J. Cell. Biochem. Suppl. 30-31:51-61.

11. Ducy, P., et al. 2000. Leptin inhibits bone formation through a hypothalamic relay: a central control of bone mass. Cell. 100:197-207.

12. Potts, J.T., Jr., and Jüppner, H. 1998. Parathyroid hormone and parathyroid hormone-related peptide in calcium homeostasis, bone metabolism, and bone development: the proteins, their genes, and receptors. In Metabolic bone disease and clinically related disorders. L.V. Avioli and S.M. Krane, editors. Academic Press. San Diego, California, USA. 52-94.

13. Yasuda, H., et al. 1998. Osteoclast differentiation factor is a ligand for osteoprotegerin/osteoclastogenesis-inhibitory factor and is identical to TRANCE/RANKL. Proc. Natl. Acad. Sci. USA. 95:3597-3602.

14. Hsu, H., et al. 1999. Tumor necrosis factor receptor family member 
RANK mediates osteoclast differentiation and activation induced by osteoprotegerin ligand. Proc. Natl. Acad. Sci. USA. 96:3540-3545.

15. Suda, T., et al. 1999. Modulation of osteoclast differentiation and function by the new members of the tumor necrosis factor receptor and ligand families. Endocr. Rev. 20:345-357.

16. Hock, J.M., Gera, I., Fonseca, J., and Raisz, L.G. 1988. Human parathyroid hormone-(1-34) increases bone mass in ovariectomized and orchidectomized rats. Endocrinology. 122:2899-2904.

17. Gunness-Hey, M., and Hock, J.M. 1989. Loss of the anabolic effect of parathyroid hormone on bone after discontinuation of hormone in rats. Bone. 10:447-452.

18. Bringhurst, F.R., et al. 1993. Cloned, stably expressed parathyroid hormone $(\mathrm{PTH}) / \mathrm{PTH}$-related peptide receptors activate multiple messenger signals and biological responses in LLC-PK1 kidney cells. Endocrinology. 132:2090-2098

19. Takasu, H., Guo, J., and Bringhurst, F.R. 1999. Dual signaling and ligand selectivity of the human PTH/PTHrP receptor. J. Bone Miner. Res. 14:11-20.

20. Fox, J., Miller, M.A., Stroup, G.B., Nemeth, E.F., and Miller, S.C. 1997 Plasma levels of parathyroid hormone that induce anabolic effects in bone of ovariectomized rats can be achieved by stimulation of endogenous hormone secretion. Bone. 21:163-169.

21. Qi, H., Li, M., and Wronski, T.J. 1995. A comparison of the anabolic effects of parathyroid hormone at skeletal sites with moderate and severe osteopenia in aged ovariectomized rats. J. Bone Miner. Res. 10:948-955.

22. Hodsman, A.B., et al. 1999. The addition of a raloxifene analog (LY117018) allows for reduced PTH(1-34) dosing during reversal of osteopenia in ovariectomized rats. J. Bone Miner. Res. 14:675-679.

23. Slovik, D.M., et al. 1986. Restoration of spinal bone in osteoporotic men by treatment with human parathyroid hormone (1-34) and 1,25-dihydroxyvitamin D. J. Bone Miner. Res. 1:377-381.

24. Lane, N.E., Thompson, J.M., Strewler, G.J., and Kinney, J.H. 1995. Intermittent treatment with human parathyroid hormone (hPTH[1-34]) increased trabecular bone volume but not connectivity in osteopenic rats. J. Bone Miner. Res. 10:1470-1477.

25. Lindsay, R., et al. 1997. Randomised controlled study of effect of parathyroid hormone on vertebral-bone mass and fracture incidence among postmenopausal women on oestrogen with osteoporosis. Lancet. 350:550-555.

26. Finkelstein, J.S., and Arnold, A.L. 1999. Increases in bone mineral density after discontinuation of daily human parathyroid hormone and gonadotropin-releasing hormone analog administration in women with endometriosis. J. Clin. Endocrinol. Metab. 84:1214-1219.

27. Wu, H., et al. 1990. Generation of collagenase-resistant collagen by sitedirected mutagenesis of murine pro 1 (I) collagen gene. Proc. Natl. Acad. Sci. USA. 87:5888-5892.

28. Wu, H., Liu, X., and Jaenisch, R. 1994. Double replacement: strategy for efficient introduction of subtle mutations into the murine Col1-a1 gene by homologous recombination in embryonic stem cells. Proc. Natl. Acad. Sci. USA. 91:2819-2823.

29. Liu, X., et al. 1995. A targeted mutation at the known collagenase cleavage site in mouse type I collagen impairs tissue remodeling. J. Cell Biol. 130:227-237.

30. Zhao, W., Byrne, M.H., Boyce, B.F., and Krane, S.M. 1999. Bone resorption induced by parathyroid hormone is strikingly diminished in collagenase-resistant mutant mice. J. Clin. Invest. 103:517-524.

31. Montgomery, A.M.P., Reisfeld, R.A., and Cheresh, D.A. 1994. Integrin $\alpha_{v} \beta_{3}$ rescues melanoma cells from apoptosis in three-dimensional dermal collagen. Proc. Natl. Acad. Sci. USA. 91:8856-8860.

32. Eliceiri, B.P., and Cheresh, D.A. 1999. The role of $\alpha_{v}$ integrins during angiogenesis: insights into potential mechanisms of action and clinical development. J. Clin. Invest. 103:1227-1230.

33. Gavrieli, Y., Sherman, Y., and Ben-Sasson, S.A. 1992. Identification of programmed cell death in situ via specific labeling of nuclear DNA fragmentation. J. Cell Biol. 119:493-501.

34. Otterness, I.G., et al. 1999. Detection of collagenase-induced damage of collagen by $9 \mathrm{~A} 4$, a monoclonal C-terminal neoepitope antibody. Matrix Biology. 19:331-341.

35. Gross, J. 1981. An essay on biological degradation of collagen. In Cell biology of the extracellular matrix. E.D. Hay, editor. Plenum Press. New York, New York, USA. 217-258.

36. Krane, S.M., et al. 1996. Different collagenase gene products have different roles in degradation of type I collagen. J. Biol. Chem. 271:28509-28515.

37. Yates, A.J.P., et al. 1988. Effects of a synthetic peptide of a parathyroid hormone-related protein on calcium homeostasis, renal tubular calcium reabsorption, and bone metabolism in vivo and in vivo in rodents. $J$. Clin. Invest. 81:932-938.

38. Walker, D.G., Lapière, C.M., and Gross, J. 1964. A collagenolytic factor in rat bone promoted by parathyroid extract. Biochem. Biophys. Res. Commun. 15:397-402.
39. Sakamoto, S., Sakamoto, M., Goldhaber, P., and Glimcher, M.J. 1975. Collagenase and bone resorption: isolation of collagenase from culture medium containing serum after stimulation of bone resorption by addition of parathyroid hormone extract. Biochem. Biophys. Res. Commun. 63:172-178

40. Quinn, C.O., et al. 1990. Rat collagenase. Cloning, amino acid sequence comparison, and parathyroid hormone regulation in osteoblastic cells. J. Biol. Chem. 265:22342-22347.

41. Henriet, P., Rousseau, G.G., and Eeckhout, Y. 1992. Cloning and sequencing of mouse collagenase cDNA. Divergence of mouse and rat collagenases from the other mammalian collagenases. FEBS Lett. 310:175-178.

42. Freije, J.M.P., et al. 1994. Molecular cloning and expression of collagenase-3, a novel human matrix metalloproteinase produced by breast carcinomas. J. Biol. Chem. 269:16766-16773.

43. Nagase, H., and Woessner, J.F., Jr. 1999. Matrix metalloproteinases. J. Biol. Chem. 274:21491-21494.

44. Partridge, N.C., et al. 1987. Hormonal regulation of the production of collagenase and a collagenase inhibitor activity by rat osteogenic sarcoma cells. Endocrinology. 120:1956-1962.

45. Selvamurugan, N., Chou, W.Y., Pearman, A.T., Pulumati, M.R., and Partridge, N.C. 1998. Parathyroid hormone regulates the rat collagenase-3 promoter in osteoblastic cells through the cooperative interaction of the activator protein-1 site and the runt domain binding sequence. J. Biol. Chem. 273:10647-10657.

46. Mattot, V., et al. 1995. Expression of interstitial collagenase is restricted to skeletal tissue during mouse embryogenesis. J. Cell Sci. 108:529-535.

47. Fuller, K., and Chambers, T.J. 1995. Localisation of mRNA for collagenase in osteocytic, bone surface and chondrocytic cells but not osteoclasts. J. Cell Sci. 108:2221-2230.

48. Gack, S., et al. 1995. Expression of interstitial collagenase during skeletal development of the mouse is restricted to osteoblast-like cells and hypertrophic chondrocytes. Cell Growth Differ. 6:759-767.

49. Ståhle-Bäckdahl, M., et al. 1997. Collagenase-3 (MMP-13) is expressed during human fetal ossification and re-expressed in postnatal bone remodeling and in rheumatoid arthritis. Lab. Invest. 76:717-728.

50. Lanske, B., et al. 1998. The parathyroid hormone (PTH)/PTH-related peptide receptor mediates actions of both ligands in murine bone. Endocrinology. 139:5194-5204.

51. Cole, A.A., et al. 1996. Chondrocyte matrix metalloproteinase-8. Human articular chondrocytes express neutrophil collagenase. J. Biol. Chem. 271:11023-11026.

52. Apte, S.S., Fukai, N., Beier, D.R., and Olsen, B.R. 1997. The matrix metalloproteinase-14 (MMP-14) gene is structurally distinct from other MMP genes and is co-expressed with the TIMP-2 gene during mouse embryogenesis. J. Biol. Chem. 272:25511-25517.

53. Ohuchi, E., et al. 1997. Membrane type 1 matrix metalloproteinase digests interstitial collagens and other extracellular matrix macromolecules. J. Biol. Chem. 272:2446-2451.

54. Holmbeck, K., et al. 1999. MT1-MMP-deficient mice develop dwarfism, osteopenia, arthritis, and connective tissue disease due to inadequate collagen turnover. Cell. 99:81-92.

55. Zhou, Z., et al. 2000. Impaired endochondral ossification and angiogenesis in mice deficient in membrane-type matrix metalloproteinase I. Proc. Natl. Acad. Sci. USA. 97:4052-4057.

56. Werb, Z. 1997. ECM and cell surface proteolysis: regulating cellular ecology. Cell. 91:439-442.

57. Staatz, W.D., Rajpara, S.M., Wayner, E.A., Carter, W.G., and Santaro, S.A. 1989. The membrane glycoprotein Ia-IIa (VLA-2) complex mediates the $\mathrm{Mg}^{+2}$-dependent adhesion of platelets to collagen. J. Cell Biol. 108:1917-1924.

58. Pilcher, B.K., et al. 1997. The activity of collagenase- 1 is required for keratinocyte migration on type I collagen matrix. J. Cell Biol. 137:1445-1457.

59. Brooks, P.C., et al. 1996. Localization of matrix metalloproteinase MMP2 to the surface of invasive cells by interaction with integrin $\alpha_{v} \beta_{3}$. Cell. 85:683-693.

60. Strömblad, S., Becker, J.C., Yebra, M., Brooks, P.C., and Cheresh, D.A 1996. Suppression of P53 activity and $\mathrm{P} 21^{\mathrm{WAF} 1 / \mathrm{CIP} 1}$ expression by vascular integrin $\alpha_{v} \beta_{3}$. J. Clin. Invest. 98:426-433.

61. Pfeilschifter, J., and Mundy, G.R. 1987. Modulation of type $\beta$ transforming growth factor activity in bone cultures by osteotropic hormones. Proc. Natl. Acad. Sci. USA. 84:2024-2028.

62. Bonewald, L.F., et al. 1997. Effects of retinol on activation of latent transforming growth factor- $\beta$ by isolated osteoclasts. Endocrinology. 138:657-666.

63. Burger, E.H., and Klein-Nulend, J. 1999. Mechanotransduction in bone: role of the lacuno-canalicular network. FASEB J. 13(Suppl.):S101-S112.

64. Jilka, R.L., et al. 1999. Increased bone formation by prevention of osteoblast apoptosis with parathyroid hormone. J. Clin. Invest. 104:439-446. 